\title{
Photoluminescence of a single InAs quantum dot molecule under applied electric field
}

\author{
I. Shtrichman, C. Metzner, B. D. Gerardot, W. V. Schoenfeld, and P. M. Petroff \\ Materials Department, University of California, Santa Barbara, CA 93106, USA
}

(November 4, 2018)

We study the electronic coupling between two vertically stacked InAs quantum dots, which are embedded in the center of a n-i-n structure. We use a micro-photoluminescence setup to optically isolate a single quantum dot pair and measure the time-averaged photoluminescence under an applied vertical electric field. We find that field tunable coupling between excited states of the two quantum dots leads to charge transfer from one dot to the other. We model the spectra including simultaneously the field dependent charge transfer and exciton capture rates, and the many-body spectra of the quantum dot molecule for different carrier configurations.

PACS: 73.21.La, 78.55.Cr, 78.67.Hc

The emerging field of quantum computation has attracted great interest over the last few years [1]. Various theoretical schemes were proposed for the implementation of quantum bits (qubits) and quantum gates, using semiconductor quantum dots (QDs) 2. Specifically, the vertically stacked double QD system was suggested to host a single [3, 4] or two qubits [5]. One can then control the coherent two-level system (qubit) with short optical pulses [3,5], by an applied electric field [4,6.7], or by a magnetic field [6]. Once the basic quantum operation in such a system is achieved, scaling up to high-density self-assembled ordered arrays of these units should be feasible [8]. A necessary step towards realization of a single qubit in a QD pair is to achieve electronic (wavefunction) coupling between the two dots. In recent years, several attempts have been made in this direction by comparing samples with different inter-dot spacing [9.10].

Clearly, the coupling between the two QDs in the pair is highly sensitive to their relative energy levels. These energies are fixed for each QD by its dimensions and material composition, which are hard to control, especially in the technologically important type of self assembled QDs. However, by varying an electric field across the QD pair one can tune the electronic states of the two QDs into and out of resonance. This method allows one to investigate the electronic coupling between the two dots in a single, specific QD molecule, thus avoiding the difficulty of comparing different molecules from various samples with each other.

In this work we study the photoluminescence (PL) spectra of two vertically stacked QDs as a function of excitation intensity and external electric field. We compare the spectra of a single dot (QD atom), an electronically uncoupled QD pair and a coupled QD molecule.
For the QD molecule we find that the two dots have a large ground state energy difference and that coupling occurs between their excited states. By tuning the electric field across the molecule we control the transfer of charge between the two dots, which is revealed in the time-averaged emission spectra, in similarity to recent works on charged single QDs 11.

Three sample structures were grown by molecular beam epitaxy (MBE) on GaAs substrates. The samples contain an intrinsic layer embedded between two n-doped GaAs layers $\left(100 \mathrm{~nm}\right.$ thickness, $1 \cdot 10^{19} \mathrm{~cm}^{-3} \mathrm{Si}$ dopant density), which serve as the front and back electrodes of the device (n-i-n structure). The intrinsic layer contains either a single InAs QD layer (sample A), or two vertically stacked strain coupled QD layers separated by a GaAs spacer of $\mathrm{d}=15 \mathrm{~nm}$ (sample B) or $\mathrm{d}=4.5 \mathrm{~nm}$ (sample C). The QD layers are located in the center of a thick GaAs layer $(200 \mathrm{~nm})$, which in turn is surrounded by two superlattice barriers (20 periods of $0.5 / 0.5 \mathrm{~nm}$ $\mathrm{GaAs} / \mathrm{AlAs}$ ) to reduce current flow through the device.

The self-assembled QDs were grown using the partially covered island technique [12]. The lens shaped QDs in the first grown layer have a height of $\approx 3 \mathrm{~nm}$ and a lateral size of $\approx 50 \mathrm{~nm}$. Due to the strain field from the first QDs layer, the dots in the second InAs layer tend to nucleate directly above the dots in the first layer 13,14 . For a QD layer separation of $15 \mathrm{~nm}$ it was shown that the pairing probability is higher than 0.9 , while for $4.5 \mathrm{~nm}$ separation the probability is close to 1 [13. The growth of the second layer QDs is strongly influenced by the strain from the first layer, resulting large size and composition difference between the two dots in the pairs 13 15.

The samples were not rotated during the InAs deposition and therefore a gradient in the QD density was formed across the wafers. A low QD density part of each of the three samples was processed by conventional lithography. A shallow mesa was chemically etched to define the device area and isolate the top conducting ndoped GaAs layer. Next, a semi-transparent $6 \mathrm{~nm}$ thick Pt layer was evaporated on the surface to provide the top gate. A $400 \mu \mathrm{m}$ square gold frame was then deposited on top of the Pt layer to serve as the front contact to the sample. An ohmic contact to the back electrode was accomplished by deposition and rapid thermal anneal of an AuGeNi alloy. We note that the devices are rectifying at low temperatures and in the relevant range of applied voltages the current is well below $100 \mu \mathrm{A}$.

For PL measurements of the high QD density parts of the wafers $\left(\approx 10^{10} \mathrm{~cm}^{-2}\right)$ we used a closed cycle Helium 
cryostat to cool down the samples to a temperature of $3 \mathrm{~K}$. A HeNe laser was used for the excitation, and the PL signal was collected and dispersed by a $0.19 \mathrm{~m}$ single monochromator followed by a liquid nitrogen cooled CCD detector. The spectral resolution was limited to $0.6 \mathrm{meV}$ (FWHM). For micro-PL measurements of the low QD density parts of the wafers $\left(\approx 5 \cdot 10^{6} \mathrm{~cm}^{-2}\right)$ we used a Helium flow cryostat to cool down the samples to $15 \mathrm{~K}$. An objective lens, used for both tight-focus excitation and luminescence collection, was located inside the cryostat, enabling a short working distance from the sample surface $(1.3 \mathrm{~mm})$. In order to achieve high spatial resolution for the collected $\mathrm{PL}$ signal in addition to the excitation, a $1 \mu \mathrm{m}$ object was defined on the sample by imaging it on a $50 \mu \mathrm{m}$ pinhole, thus filtering out any light coming from outside the object light cone. The spatially filtered luminescence was then dispersed and detected by the same setup as in the conventional PL. The overall spatial resolution was measured to be $1.1 \mu \mathrm{m}$ (FWHM) at $843 \mathrm{~nm}$, while the average distance between the dots was about $5 \mu \mathrm{m}$, enabling us to optically isolate and study a single QD pair.

When an electric field is varied at fixed optical excitation intensity, the effective rate of exciton capture into the QDs changes drastically due to field-induced carrier escape processes. For the micro-PL measurements under applied DC electric field we have significantly reduced this effect by using a continues wave Ti:Sapphire laser tuned to an energy below the GaAs barrier (1.47 eV), thus exciting only the InAs wetting layers (WLs).

We note that for each of the three samples we studied in detail at least 3 different QD pairs (or dots in sample A), which show the same qualitative behavior for each sample, although at slightly different energies.

In Fig.1 we display ensemble PL spectra of all three samples for different excitation intensities, at zero applied voltage. Fig.1 a shows the luminescence from a single layer of QDs (sample A), which at low excitation intensity peaks at $1.258 \mathrm{eV}$ and shows an inhomogeneous broadening of $50 \mathrm{meV}$ (FWHM). At high excitation intensities several excited states gradually appear in the spectra, separated by $\approx 40 \mathrm{meV}$. The luminescence from the WL emerges at $1.447 \mathrm{eV}$. For samples B (Fig.1 $\mathrm{b}$ ) and $\mathrm{C}$ (Fig.11 c) we identify the two peaks, which dominate the spectrum at the lowest excitation powers, as the inhomogeneously broadened luminescence from the two QD layers. The low-energy peak (QD1) has about the same energy as the single QD layer of sample A. We therefore relate this peak to the first grown (seed) layer of QDs in samples B and C. The high-energy peak (QD2) is assigned to the second, strain coupled layer of QDs. We attribute the separation of $\approx 60 \mathrm{meV}(90 \mathrm{meV})$ between the QDs ground states in Fig.1 b (1 $1 \mathrm{c}$ ) to the two dots unintentional size and composition differences, and to the asymmetric strain field that they induce on each other. Several excited states of QD1 and QD2, separated by $40 \mathrm{meV}$ and $25 \mathrm{meV}$, respectively, are observed in the spectra as the excitation power increases. We note that it is not necessary for the two QDs to have exactly the same ground state energies in order to achieve coupling between these states 16. However, in our case, where the two ground states are separated by $90 \mathrm{meV}$ (for $\mathrm{d}$ $=4.5 \mathrm{~nm}$ ), the tunnel coupling between these states is negligible 17.

A clear difference between samples B and C is apparent from Fig.11: In sample B the PL intensity ratio of QD1 to QD2 is fixed at low excitation intensities 18 . In contrast, in sample $\mathrm{C}$ this ratio changes as the excitation density increases. In Fig.1 d-f (samples A-C, respectively) we show the spectrally integrated PL intensity of the different states of QD1 and QD2 as a function of the excitation intensity. The various states are resolved using a multi-Gaussian fit to each of the PL spectra of Fig. 11ac. We find that in all three samples the QD1 ensemble ground state PL grows linearly with the excitation intensity over almost 6 orders of magnitude, due to gradual filling of the many dots in the ensemble. This is also the case for the QD2 ensemble ground state in sample B. In contrast, the ground state of QD2 in sample $\mathrm{C}$ saturates at much lower intensities, indicating that in this sample the two QDs are electronically coupled. Since QD2 luminescence saturates while QD1 luminescence continues to grow linearly, we deduce that one type of charge carrier is transferred from QD2 to QD1, while the opposite charge remains in QD2 (thus forming an indirect exciton in the molecule). The fact that luminescence from the ground state of QD2 is apparent at low excitation intensities suggests that this charge transfer takes place between excited states of the two dots. We note that the qualitatively different behavior of QD1 and QD2 in the ensemble PL spectra of samples $\mathrm{B}$ and $\mathrm{C}$ is reproduced also in micro-PL measurements of single QD pairs (see for instance Fig.2a, below).

In the micro-PL spectra of a single QD (sample A) we observe, as the excitation intensity is raised, a number of sharp peaks in the spectra in agreement with previous works 19,20. When varying the applied voltage at constant photo-excitation intensity, we find that the peaks in the PL spectra change their relative intensities due to a change in the effective exciton capture rate into the dot (not shown). This effect is due to tunneling of carriers out of the InAs layer and into the thick GaAs surroundings at high enough fields. We find that flat bands condition, in which the capture rate into the InAs layer is maximized, is achieved at $0.2 \mathrm{~V}$. This result is also in agreement with photocurrent measurements of the device. Due to our limited spectral resolution we see no spectral shift related to the spatially direct Stark effect, which in the relevant range of electric fields is expected to be on the order of few hundreds $\mu \mathrm{eV} 21$.

In the micro-PL spectrum of a single pair in sample $\mathrm{B}$ we observe the spectral signature of two independent QDs, which are separated by $\approx 80 \mathrm{meV}$ (not shown). Under applied electric field the spectra from this electronically uncoupled QD pair strongly resemble the single QD results. 
This behavior is in contrast with the results from a QD molecule in sample $\mathrm{C}$, as displayed in Fig.2. At zero applied voltage (Fig.2a) and low excitation intensity, QD1 and QD2 are clearly seen in the spectrum and are separated by $\approx 100 \mathrm{meV}$. The luminescence from QD2 is broad, probably due to simultaneous recombination from the ground and excited states of this dot (compare to the ensemble case, Fig.11c). At high excitation intensities QD2 disappears from the spectrum and the excited states of QD1 emerge gradually.

In Fig.2b we show the high-resolution spectra of QD1 for different applied voltages. We note that several peaks appear and disappear in the S shell luminescence of QD1 while varying the electric field. By measuring the $\mathrm{PL}$ spectrum as a function of the excitation intensity at various applied voltages (not shown), we identify the transition energy of a single neutral exciton $\left(1 X_{S}\right)$ at 1.2595 $\mathrm{eV}$, and of a neutral bi-exciton $\left(2 X_{S}\right)$ at $-2.1 \mathrm{meV}$ relative to $1 X_{S}$ (these transitions are marked by dotted lines in Fig.2 $2 \mathrm{~b}$ ). In the $-0.4 \mathrm{~V}$ spectrum we resolve additional two lines, at $-3.2 \mathrm{meV}$ and $-5.7 \mathrm{meV}$ relative to $1 X_{S}$. In the $-0.2 \mathrm{~V}$ spectrum a shoulder appears at the lowenergy part of the $\mathrm{S}$ shell spectrum. We relate these lines and shoulder to recombination of an $\mathrm{S}$ shell exciton in the presence of charged multi-exciton complexes. The above $\mathrm{S}$ shell picture then almost symmetrically reverses at positive voltages. In addition to the $\mathrm{S}$ shell luminescence, a broad $\mathrm{P}$ shell peak emerges at $\approx 1.296$ $\mathrm{eV}$ and is most intense at a voltage of $0.2 \mathrm{~V}$. As mentioned above, this behavior is due to flat bands condition at this voltage, in which the effective exciton capture rate of the QD molecule is maximized. We relate the broad $\mathrm{P}$ shell peak to luminescence from neutral and charged tri-exciton states, which are not resolved in this case.

The evolution of the steady-state micro-PL spectra in Fig.2p can be explained by a combination of two voltagedependent effects: First, a change in the neutral exciton occupation numbers $X_{1}$ and $X_{2}$ in QD1 and QD2, respectively, due to a field-dependent escape rate (shown schematically in Fig.3a). We estimate that the average exciton occupation $\bar{X}_{1}$ of QD1 drops from $\approx 3$ at voltage $\mathrm{U}=0.2 \mathrm{~V}$ to $\approx 1$ at $\mathrm{U}=0.8 \mathrm{~V}$ (dash line in Fig. $\$ \mathrm{~b}$ ). Second, a change in the probability of single carrier transfer between the QDs, which has a broad maximum around $\mathrm{U}=0 \mathrm{~V}$ and becomes negligible at $\mathrm{U}= \pm 0.8 \mathrm{~V}$ (solid line in Fig. $3 \mathrm{~b}$ ). By comparing with theoretical PL-spectra (see below), calculated for different charging scenarios, we conclude that it is an electron rather than a hole that hops from QD2 to QD1. Whenever an electron transfer occurs, a spatially indirect electron-hole pair is formed in the QD molecule, and the resulting charge dipole prevents further transfer (Coulomb blockade effect). Thus, the number $Y$ of indirect electron-hole pairs is restricted to 0 or 1 in our case.

In order to support our hypothesis we compare the micro-PL spectra of Fig.2b to a numerical simulation, the details of which will be published elsewhere 22. In our model we describe the confinement potential of the
QD molecule in the growth direction as an asymmetric double quantum well, and in the lateral directions as a rotationally symmetric parabolic well. Coulomb interaction effects are treated by direct diagonalization (configuration interaction method [23]) of the respective manybody Hamiltonian, which is defined by the momentary population $\left(X_{1}, Y, X_{2}\right)$ of the $\mathrm{QD}$ molecule. We assume complete energy and spin relaxation of the carriers prior to each recombination event. Using the resulting correlated many-body states, we compute the PL-emission spectrum $I\left(\hbar \omega, X_{1}, Y, X_{2}\right)$ for each relevant population. The time-integrated spectrum $I_{a v}(\hbar \omega, U)$ is calculated by averaging $I\left(\hbar \omega, X_{1}, Y, X_{2}\right)$ over all possible populations, weighted by their voltage-dependent formation probability $P\left(X_{1}, Y, X_{2}, U\right)$. This probability is determined by the lifetimes of the exciton complexes, the escape rates of excitons from the WL (modeled as a tunneling process out of a quantum well), and the effective rate of electron inter-dot transfer (modeled as an acousticphonon-assisted hopping process with resonant tunneling enhancement and including level broadening due to the statistical population fluctuations).

In Fig. 1 we display the calculated spectra at different applied voltages (we assume a linear drop of the voltage across the intrinsic layer of the sample). The main peaks in the extreme voltages spectra $( \pm 0.8 \mathrm{~V})$ are related to several possible optical transitions from the various neutral multi-exciton configurations 20,23], in QD1: $1 X_{S}$ $(1.2599 \mathrm{eV}), 2 X_{S}(1.2577 \mathrm{eV}), 3 X_{S}(1.2484 \mathrm{eV}, 1.2582$ $\mathrm{eV}), 3 X_{P}(1.291 \mathrm{eV})$. The spectrum at $0 \mathrm{~V}$ is dominated by configurations of negatively charged multi-excitons: $1 X_{S}^{-}(1.2574 \mathrm{eV}), 2 X_{S}^{-}(1.2529 \mathrm{eV}), 2 X_{P}^{-}(1.2907 \mathrm{eV})$, $3 X_{S}^{-}(1.2483 \mathrm{eV}, 1.2503 \mathrm{eV}, 1.2517 \mathrm{eV}), 3 X_{P}^{-}(1.2853$ $\mathrm{eV}, 1.2928 \mathrm{eV})$. The calculated spectra reproduce well the main features of the experimental spectra (Fig.2pb). A similar calculation, which does not take into account charge transfer between the dots, leads to a qualitatively different spectral behavior [22].

In summary, we have measured the time-averaged PL of single vertically stacked InAs QD pairs under applied electric field. For the closely spaced QD pair (molecule) we find that coupling between excited states of the two dots leads to field tunable electron transfer from one dot to the other. Our theoretical model reproduces the experimental results by simultaneously taking into account the field-dependent carriers kinetics in the QD molecule, and the molecule's many-body spectrum for different carriers populations.

This work was supported by the ARO-DARPA grant and the Humboldt Foundation. 
[1] For a review see C. H. Bennett and D. P. DiVincenzo, Nature 404, 247 (2000).

[2] D. Loss and D. P. DiVincenzo, Phys. Rev. A 57, 120 (1998), A. Imamoglu et al., Phys. Rev. Lett. 83, 4204 (1999), X. Hu and S. Das Sarma, Phys. Rev. A 61, 062301 (2000).

[3] X. Q. Li and Y. Arakawa, Phys. Rev. A 63, 012302 (2000).

[4] A. Balandin and K. L. Wang, Superlatt. Microstruct. 25, 509 (1999).

[5] E. Biolatti et al., Phys. Rev. Lett. 85, 5647 (2000).

[6] G. Burkard, G. Seelig, and D. Loss, Phys. Rev. B 62, 2581 (2000).

[7] A. Barenco et al., Phys. Rev. Lett. 74, 4083 (1995).

[8] H. Lee et al., Appl. Phys. Lett. 78, 105 (2001), G. Springholz et al., Science 282, 734 (1998).

[9] G. Schedelbeck et al., Science 278, 1792 (1997).

[10] M. Bayer et al., Science 291, 451 (2001).

[11] A. Hartmann et al., Phys. Rev. Lett. 84, 5648 (2000), R. J. Warburton et al., Physica E 9, 124 (2001), D. V. Regelman et al., Proc. 25th Int. Conf. Phys. Semicond., Osaka 2000, Edited by N. Miura and T. Ando(SpringerVerlag, 2001).

[12] J. M. Garcia et al., Appl. Phys. Lett. 72, 3172 (1998).

[13] Q. Xie et al., Phys. Rev. Lett. 75, 2542 (1995).

[14] G. S. Solomon et al., Phys. Rev. Lett. 76, 952 (1996).

[15] R. Heitz et al., Phys. Rev. B, 58, R10151 (1998).

[16] M. Korkusinski and P. Hawrylak, Phys. Rev. B 63, 195311 (2001).

[17] R. Ferreira and G. Bastard, Rep. Prog. Phys. 60, 345 (1997).

[18] The PL intensity ratio QD1/QD2 varies for different samples with nominally the same structure, and may be related to some extrinsic, non-radiative recombination channels.

[19] E. Dekel et al., Phys. Rev. Lett. 80, 4991 (1998), L. Landin et al., Science 280, 262 (1998).

[20] E. Dekel et al., Phys. Rev. B 61, 11009 (2000).

[21] S. Raymond et al., Phys. Rev. B 58, R13415 (1998), H. Htoon et al., Appl. Phys. Lett. 76, 700 (2000).

[22] I. Shtrichman et al., to be published in Physica E.

[23] L. Jacak, P. Hawrylak, and A. Wojs, Quantum dots (Springer-Verlag, Berlin, 1998). 

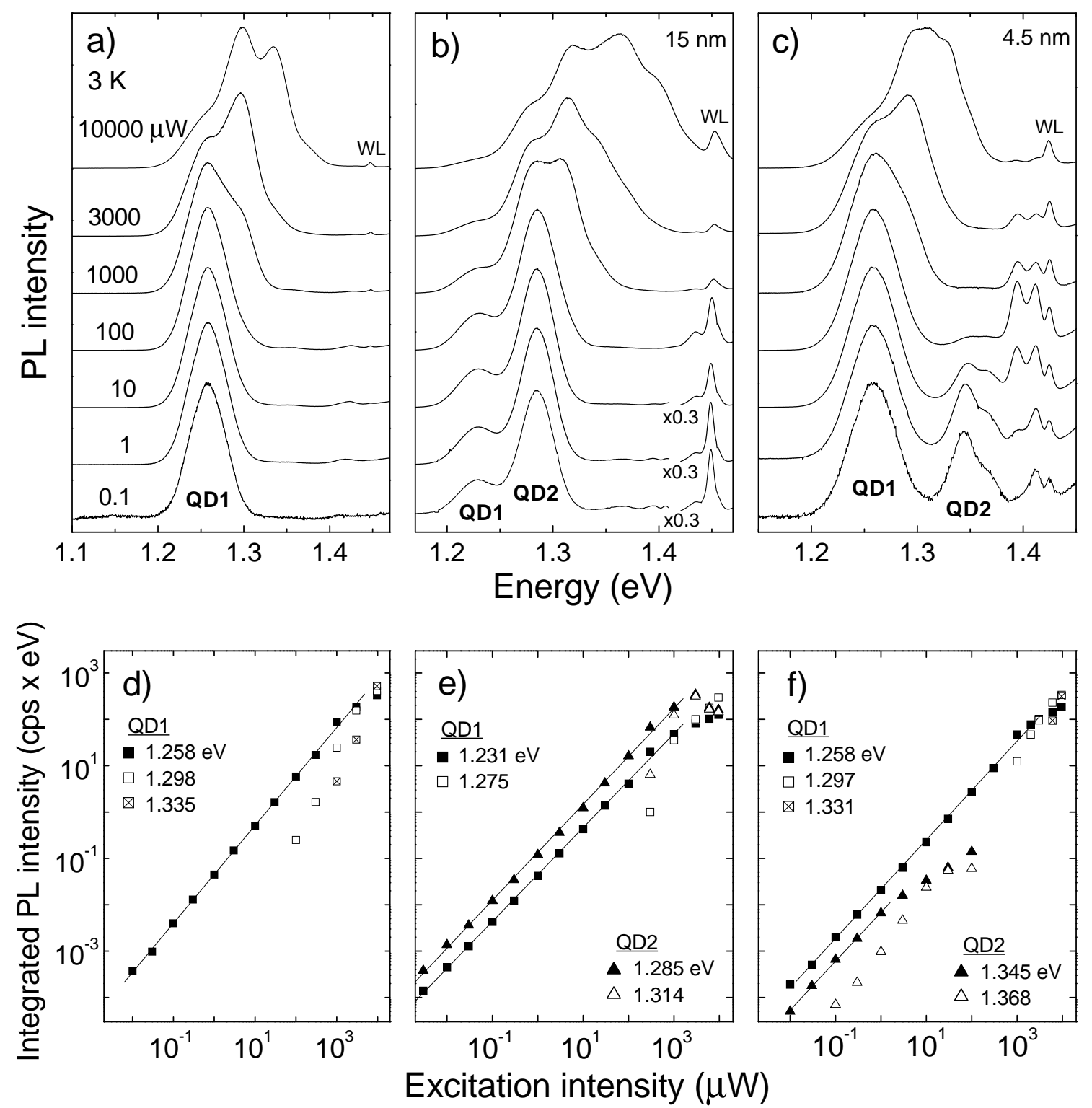

FIG. 1. a-c) PL spectra from the high QD density part of samples A, B and C, respectively, for different excitation intensities. The spectra are vertically displaced for clarity. The excitation energy is $1.96 \mathrm{eV}$, and $1 \mu \mathrm{W}$ corresponds to a density of 0.02 $\mathrm{W} / \mathrm{cm}^{2}$. d-f) Spectrally integrated intensity of the different PL peaks as a function of excitation intensity in samples A, B and $\mathrm{C}$, respectively. The solid lines are linear fits to the data. 

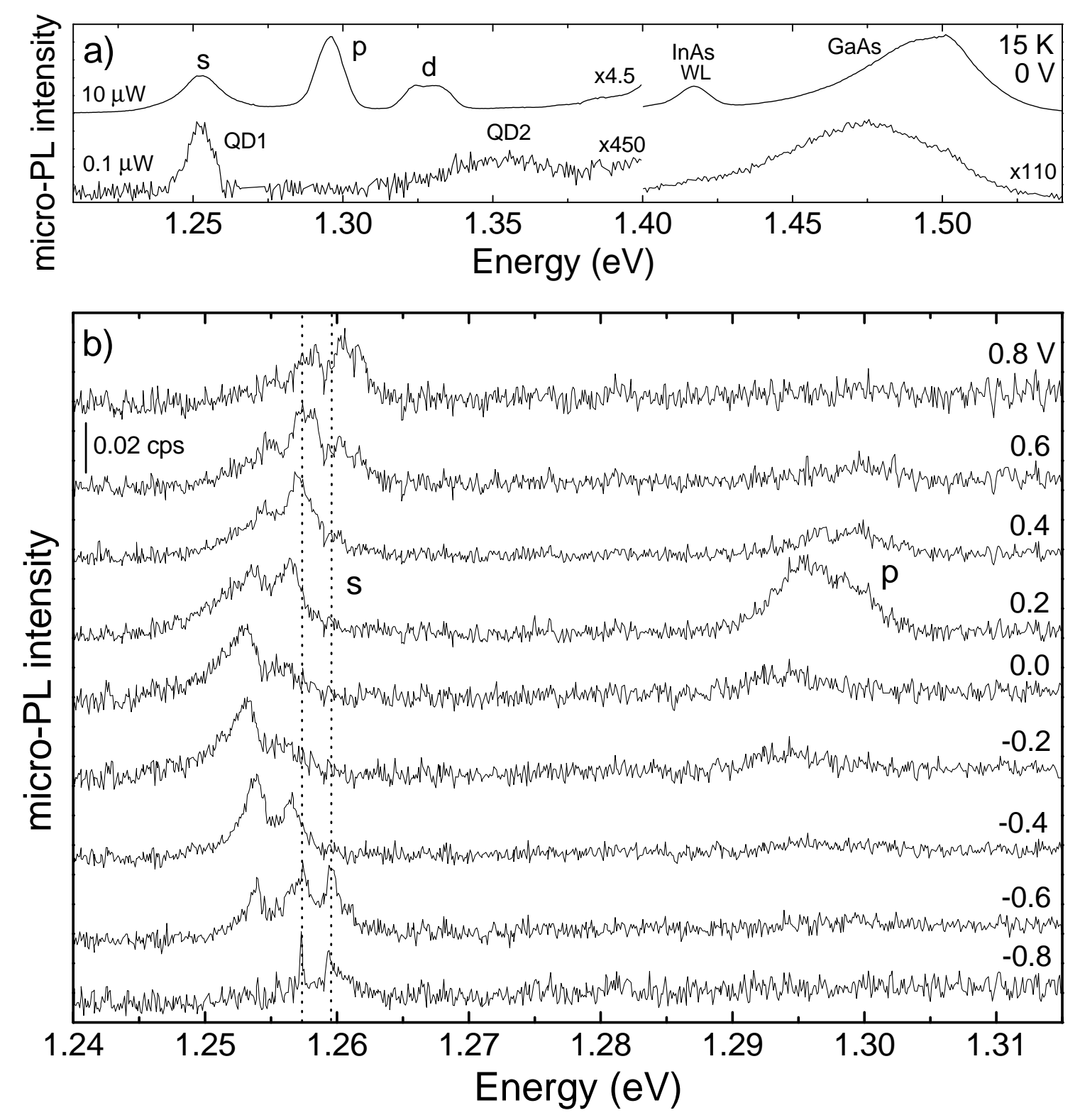

FIG. 2. Micro-PL spectra of a single coupled QD molecule (sample C). a) Low resolution spectra excited at $1.96 \mathrm{eV}$ and at zero applied voltage. b) High resolution spectra for different applied DC voltages. The excitation energy and intensity are 1.47 $\mathrm{eV}$ and $100 \mu \mathrm{W}$, respectively, and $1 \mathrm{~V}$ corresponds to an electric field of $38 \mathrm{kV} / \mathrm{cm}$. 

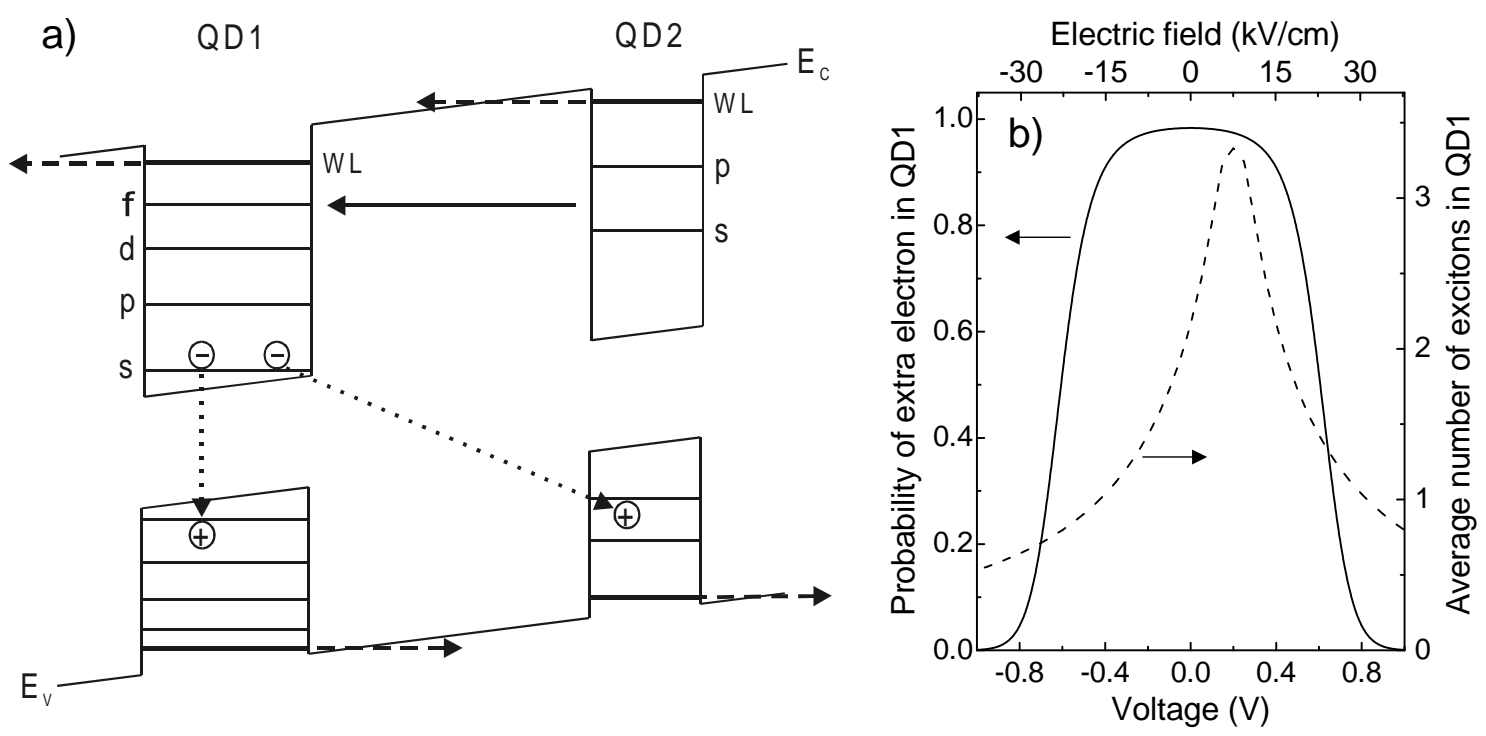

FIG. 3. a) Schematic bandstructure along the growth direction of a QD molecule under applied electric field. The arrows mark the main kinetic processes of our model. In this example QD1 is populated by a single exciton plus one extra electron, which was transferred from QD2, leaving behind a hole, i.e. $X_{1}=1, Y=1, X_{2}=0$. b) Calculated probability of extra electron (solid line, left axis), and average number $\overline{X_{1}}$ of excitons in QD1 (dash line, right axis) as a function of applied voltage. 


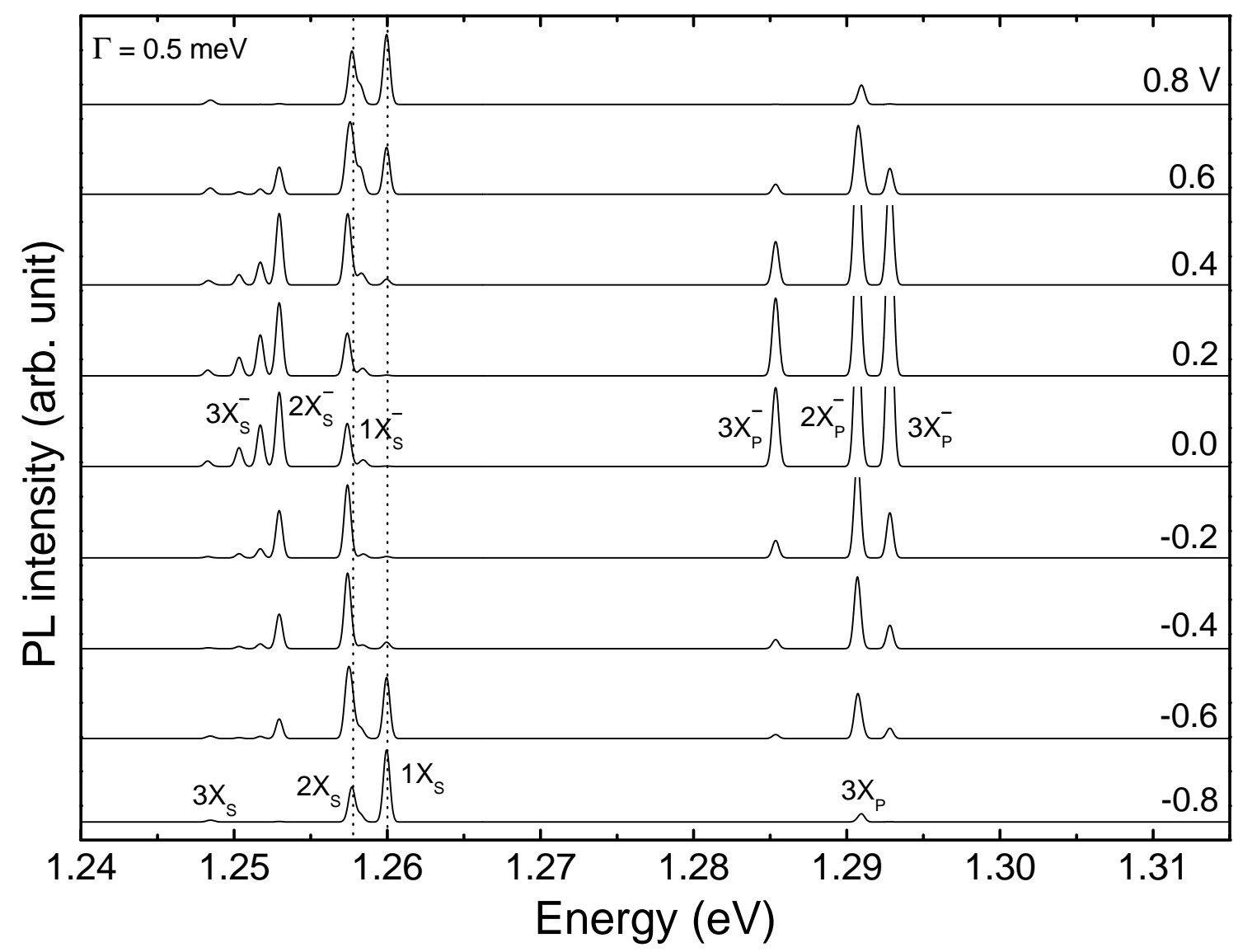

FIG. 4. Calculated PL spectra of a coupled QD molecule with inter-dot distance $d=4.5 \mathrm{~nm}$ at different applied voltages. The spectra lines are artificially broadened by $0.5 \mathrm{meV}$. 